\section{Aspectos históricos da institucionalização da atenção à saúde do adolescente no estado de São Paulo, 1970-1990}

\author{
Historical aspects of the \\ institutionalization of \\ adolescent healthcare in \\ the state of São Paulo, \\ 1970-1990
}

Lígia Bruni Queiroz

Médica da Unidade de Adolescentes do Instituto da Criança do Hospital das Clínicas da Faculdade de Medicina/ Universidade de São Paulo (USP).

Av. Dr. Enéas Carvalho de Aguiar, 647 05403-000 - São Paulo - SP - Brasil ligiabq@usp.br

José Ricardo de Carvalho Mesquita Ayres Professor titular do Departamento de Medicina Preventiva da Faculdade de Medicina/USP. Av. Dr. Arnaldo, 455, sala 2213

01246-903 - São Paulo - SP - Brasil

jrcayres@usp.br

\section{Maria Ignez Saito}

Professora livre-docente pelo Departamento de Pediatria da Faculdade de Medicina/USP.

Av. Dr. Enéas Carvalho de Aguiar, 647

05403-000 - São Paulo - SP - Brasil

maria.saito@icr.usp.br

\section{André Mota}

Coordenador do Museu Histórico

Prof. Carlos da Silva Lacaz da Faculdade de Medicina/USP.

Av. Dr. Arnaldo, 455, 4 andar

01246-903 - São Paulo - SP - Brasil

amota@museu.fm.usp.br

Recebido para publicação em fevereiro de 2011.

Aprovado para publicação em abril de 2012.
QUEIROZ, Lígia Bruni et al. Aspectos históricos da institucionalização da atenção à saúde do adolescente no estado de São Paulo, 1970-1990. História, Ciências, Saúde - Manguinhos, Rio de Janeiro, v.20, n.1, jan.-mar. 2013, p.49-66.

\section{Resumo}

Trata de aspectos históricos da atenção integral à saúde dos adolescentes no estado de São Paulo, sobretudo quanto à implantação de serviços e programas pioneiros. Contextualiza desafios, embates e dificuldades gerados no âmbito das instituições implicadas no desenvolvimento desse campo, à luz das perspectivas da medicina clínica e da saúde coletiva, tendo como pano de fundo o contexto sociopolítico brasileiro na época. Fundamentado em material documental, recupera-se a construção de núcleos de expertise e do campo da saúde do adolescente na dialética entre a construção de uma nova área de atuação médica com base na clínica e um campo de saberes e práticas da saúde coletiva com base na integralidade da atenção à saúde, de caráter interdisciplinar e intersetorial.

Palavras-chave: saúde do adolescente; história; saúde coletiva; medicina do adolescente.

\section{Abstract}

The article explores historical aspects of integral healthcare for adolescents in the state of São Paulo, particularly in regard to the implementation of pioneering services and programs. Against the backdrop of Brazil's social and political context during this period, it contextualizes the challenges, clashes, and difficulties that arose within the institutions involved in developing this field, from the perspectives of clinical medicine and of collective health. Grounded on documental material, the study re-examines the construction of groups of expertise and of the field of adolescent health care as part of the dialectical interplay between the construction of a new area of medical practice based on clinical work and a field of knowledge and practices in collective health based on integral health care of an interdisciplinary, inter-sectoral nature.

Keywords: adolescent health; history; collective health; adolescent medicine. 
$\mathrm{C}$ om o objetivo central de recuperar as dimensões históricas que configuram o contexto da atenção à saúde integral ao adolescente no estado de São Paulo entre as décadas de 1970 e 1990, o artigo busca compreendê-la à luz de suas inter-relações com a medicina clínica, representada aqui mais especificamente pela pediatria - na qual se insere a medicina de adolescentes - e pela saúde coletiva, tendo como cenário os processos históricos então vigentes no Brasil e as concepções sobre os processos de saúde/doença e cuidado em disputa no ambiente acadêmico da época.

É também objetivo deste artigo compreender a importância dada à saúde do adolescente, sobretudo nesse período, quando se discutia a efetiva prática da medicina integral, e se questionava a fragmentação do indivíduo - efeito colateral da emergência das subespecialidades médicas e da medicalização do sujeito na medicina moderna.

A delimitação temporal do estudo decorreu da importância desse período no processo de institucionalização da medicina de adolescentes nas principais faculdades de medicina do país e, particularmente, em São Paulo. Com isso, consegue-se aprofundar a compreensão do movimento histórico aqui considerado e traçar as relações entre o contexto político do país e a preocupação com a saúde do adolescente, tanto na perspectiva da clínica quanto da saúde coletiva.

De acordo com Campos (2000), a institucionalização dos saberes científicos e sua organização em práticas dá-se mediante a conformação de núcleos e campos, sendo os primeiros aglutinações de conhecimentos que demarcam a identidade de uma área de saber e de prática profissional, e os últimos, espaços de limites imprecisos em que cada disciplina e profissão busca apoio em outras para cumprir suas tarefas teóricas e práticas relativas a algum interesse compartilhado.

O conceito de campo, tal como utilizado por esse autor, embora guarde afinidades com o célebre conceito desenvolvido por Pierre Bourdieu, dele se distingue, como apontado por Campos (2000), não pelo fato de buscar traduzir com ele, e o correlato conceito de núcleo, a passagem de processos intelectuais abertos para estruturas fechadas de saberes e poderes institucionalizados em disciplinas (corpus), mas por buscar apreender a relação entre campos e núcleos (áreas disciplinares) em sua mutualidade dinâmica, no constante processo pelo qual os núcleos causam transformações e são transformados no contato com seus campos de interesse e com os demais núcleos que os constituem. É essa construção de Campos que parece melhor servir aos propósitos do presente estudo. Nesse sentido, vamos considerar aqui a atenção à saúde integral do adolescente um campo de saberes e práticas relacionado a dois núcleos disciplinares principais: o pediátrico e o sanitário. A medicina de adolescentes é, nesse quadro, a expressão da emergência do campo da atenção integral à saúde do adolescente no âmbito institucional da pediatria, assim como a saúde do adolescente é expressão de sua emergência no âmbito institucional da saúde pública. Cabe ressaltar, contudo, que é na perspectiva de um sanitarismo identificado com outro campo, chamado de saúde coletiva, que nasce o interesse pela saúde do adolescente. Por isso, utilizaremos neste texto a expressão saúde coletiva para nos referir não a esse campo em si, mas ao núcleo disciplinar que se aproxima, pela perspectiva sanitária, do campo da atenção integral à saúde do adolescente.

A trajetória histórica aqui recuperada foi estudada mediante levantamento e análise de documentos (livros, periódicos, revistas, jornais, cartas, teses acadêmicas, material de 
congressos e publicações de associações médicas) produzidos nas décadas de 1970 a 1990 acerca da institucionalização da medicina de adolescentes, em busca de relações com o momento político em questão - a ditadura militar no Brasil e os movimentos sociais a ela relacionados - e a institucionalização da medicina de adolescentes e da saúde do adolescente no estado de São Paulo.

O período histórico estudado coincide com a integração mundial e nacional da juventude como nova categoria social, politicamente ativa e culturalmente inovadora. Simultaneamente, a medicina de adolescentes ingressa num processo de institucionalização, como que acompanhando a emergência dos jovens enquanto atores sociopolíticos relevantes no cenário social. Por fim, contradições e embates políticos e acadêmicos vêm à luz exatamente no período de implantação da ditadura militar no Brasil (com o golpe de 1964 e, em 1968, a edição do ato institucional n.5, o AI-5) (Brasil, 13 dez. 1968), cujas consequências mais deletérias não deixam de ser sentidas até o final dos anos 1980 (Mattos, Swensson Junior, 2003).

São necessárias ainda algumas considerações a respeito dos conceitos medicina de adolescentes, clínica e saúde dos adolescentes. Como vimos, entende-se por medicina de adolescentes a área de atuação da pediatria que se constitui como núcleo clínico no campo da atenção à saúde do adolescente. O médico de adolescentes é aquele que foi aprovado em exame que lhe confere o título de especialista em pediatria, reconhecido e regulado pela Sociedade Brasileira de Pediatria (SBP), pela Associação Médica Brasileira (AMB) e pelo Conselho Federal de Medicina (CFM); só assim esse médico está habilitado a prestar uma segunda prova que lhe confere, desde 1998, a habilitação em medicina de adolescentes. Embora ginecologistas, psiquiatras, sanitaristas e profissionais de outras áreas possam atender adolescentes, se capacitados para tal, historicamente o título tem sido da competência da pediatria. Muitos utilizam indiscriminada e, para essa habilitação, equivocadamente o termo hebiatria como sinônimo de medicina de adolescentes.

A clínica, tal como empregada no presente estudo, refere-se a um conjunto de saberes intrinsecamente relacionado ao trabalho médico a partir do século XIX e que tem em sua raiz histórica duas ideias herdadas do positivismo: "autonomia do objeto de conhecimento por referência ao sujeito desse conhecimento e a legitimação de sua explicação causal a partir da reprodutibilidade positivamente aferível de eventos a que se referem uma vez reproduzidas as mesmas condições a que se associou a causação" (Ayres, 1990, p.142). Por saúde do adolescente entende-se uma série de ações, programas e práticas (tanto assistenciais quanto sanitárias), que integram as mais diversas áreas, como educação, cultura, esporte, trabalho e lazer, para a promoção de saúde e prevenção de agravos nessa faixa etária, considerando os adolescentes componentes de um segmento populacional com necessidades de cuidados físicos e psicoafetivos específicos.

Assim, medicina de adolescentes e saúde dos adolescentes constituíram-se historicamente pelo interesse de conhecimento e intervenção sobre sujeitos caracterizados por uma dada condição física socioculturalmente investida de significados específicos e necessidades de saúde particulares. A primeira decorrente de um olhar mais focado no indivíduo, a segunda do olhar sobre populações, ambas em relações estreitas e tensas, de diferentes modos, com a clínica em seu sentido mais tradicional. 


\section{Adolescência: concepções e origens históricas}

Do ponto de vista etário, aspecto cronológico do ciclo vital, a Organização Mundial de Saúde (OMS, 1977) considera adolescente o indivíduo entre os 10 e os 19 anos, e jovem aquele entre 15 e 24 anos. Identificam-se, assim, como adolescentes jovens os indivíduos com idade de 15 a 19 anos, e adultos jovens aqueles com idade de 20 a 24 anos. No Brasil, o Estatuto da Criança e do Adolescente (ECA) define por adolescente o indivíduo entre 12 e 18 anos de idade (Brasil, 13 jul. 1990).

Ruzany e Szwarcwald (2000) consideram adolescência (conceito que se estabelece nas publicações especializadas sobre o tema) a etapa da vida compreendida entre a infância e a idade adulta, marcada por um complexo processo de crescimento e desenvolvimento biopsicossocial.

A palavra "adolescência" é derivada do verbo latino adolescere, significando crescer ou crescer até a maturidade. "Puberdade", por sua vez, origina-se dos termos latinos pubertas e pubescere, que significam apresentar pelos no corpo e atingir a maioridade; o conceito de puberdade, portanto, refere-se às mudanças biológicas e fisiológicas associadas à maturação sexual (Muuss, 1976).

Categoria ambígua, a adolescência costuma ser tomada como identidade de um ser em transição da infância à condição de adulto, portanto, em uma condição liminar, marginal, o que o aproxima do neófito dos rituais de passagem. No meio urbano ocidental, entretanto, percebe-se um prolongamento dos limites da adolescência, em contraste com o que ocorre em sociedades ditas primitivas, nas quais essa fase de vida é marcada por intervenções bem delimitadas, denominadas ritos de passagem. ${ }^{1}$

A definição etária da adolescência proposta pela OMS, com limites amplos, que variam de 10 a 20 anos, é capaz de abranger a maioria dos eventos puberais. Já o prolongamento social do período de estudos e, com isso, a maior dependência econômica, postergam a juventude para além dos 20 anos, reduzindo-se, assim, o período de infância e adultícia, contribuindo, contudo, para tornar mais nítidas e bem demarcadas essas duas faixas etárias.

A partir da Idade Média e até o início dos tempos modernos, as crianças misturavam-se aos adultos tão logo eram consideradas capazes de dispensar a ajuda das mães ou das amas, poucos anos depois de um desmame tardio, ou seja, aproximadamente aos sete anos de idade. Ingressavam, então, na comunidade dos homens, participando, com seus amigos jovens ou idosos, dos trabalhos e dos jogos cotidianos (Ariès, 1981). Não havia adolescência, tal como hoje é concebida, até meados do século XIX, quando começa a ser reconhecida como período crítico para a existência humana.

A atual concepção de adolescência surge inicialmente na Europa, a partir da segunda metade do século XIX, e está estreitamente relacionada às mudanças econômicas, políticas e culturais geradas no bojo do processo de industrialização e pelo estabelecimento do sistema escolar obrigatório imposto pelo desenvolvimento dos países europeus. Nesse momento histórico, ampliavam-se mundialmente a demografia, a urbanização e a divisão do trabalho, donde a intensa especialização da mão de obra (Rojas, 1995).

Na segunda metade do século XX, ocorreu o que se denominou revolução cultural, atingindo a organização da família e provocando modificações na estrutura de relações entre 
os sexos e as gerações. A família nuclear, que se tornou modelo-padrão na sociedade ocidental nos séculos XIX e XX, começa a declinar durante as décadas de 1970 e 1980. São identificados uma diminuição de interesse por parte das mulheres no casamento formal, um arrefecimento do desejo de ter filhos e uma mudança de atitude com relação à aceitação da diversidade de orientação sexual. Registra-se aumento do número de pessoas vivendo solitariamente e de mães solteiras ou de lares chefiados por mulheres, tanto em países da Europa quanto nos EUA (Hobsbawm, 1997). A crise da família relaciona-se a mudanças nos valores e regras, oficiais ou não, que governam a conduta sexual, a parceria e a procriação. Tais mudanças centram-se nas décadas de 1960 e 1970.

Após a Segunda Guerra Mundial, a humanidade presenciou forte crescimento econômico, com repercussões na elevação da renda da população e no consumo de produtos industrializados. A mídia passou a exercer, nesse contexto, papel fundamental, tanto na divulgação de novos produtos quanto na veiculação de modas e tendências, destinados sobretudo aos jovens, à luz da premissa de serem eles indivíduos abertos a mudanças e inovações, e suscetíveis a influências políticas e tecnológicas modernizadoras. Tais características são identificadas e exploradas pelos meios de comunicação, que contribuíram, em larga escala, para a construção da identidade do adolescente na sociedade urbana e industrial, marcando seu ingresso no mercado de consumo.

A concepção de juventude corrente na literatura sociológica contemporânea transcende os aspectos cronológicos ou meramente biológicos. Segundo Marialice Foracchi (1972, p.160), socióloga e estudiosa do tema, "Não sendo passível de delimitação etária, a juventude representa, histórica e socialmente, uma categoria social gerada pelas tensões inerentes à crise do sistema. Sociologicamente, ela representa um modo de realização da pessoa, um projeto de criação institucional, uma alternativa nova de existência social".

A juventude também é identificada por Foracchi (1972) como força dinamizadora da vida social, atuante em sua transformação, para o que se une a outras forças sociais operantes. De um lado, sua busca de uma sociedade mais justa leva-a a emergir como porta-voz dos desfavorecidos, revelando as formas de opressão existentes; de outro, sua flexibilidade lhe permite experimentar novas alternativas e adaptar-se com relativa facilidade a modos de conduta, valores e padrões de vida anteriormente desconhecidos.

De forma análoga ao que ocorria na Europa e nos EUA nas décadas de 1960 e 1970, os jovens da América Latina começavam a se configurar como categoria social distinta, definida por uma condição específica que demarca interesses e necessidades próprios, quase totalmente desvinculados da ideia de transição.

Nos países latino-americanos, o adolescente morador da zona urbana, no período compreendido entre as décadas de 1950 e 1980, experimentou profundas transformações em sua maneira de agir, pensar e sentir, em consequência da ampliação do período de escolarização e do gerenciamento do tempo livre. Houve amplo acesso de adolescentes e jovens, oriundos das camadas médias e baixas da população, à escola, despontando assim para eles, um horizonte otimista, com perspectivas de mobilização social e melhoria das condições de vida por meio da escolarização e de novas oportunidades de trabalho.

A expansão da educação, que legitimou a 'moratória social' do adolescente no papel de estudante, somada à massificação da televisão e ao crescimento de poderosas indústrias 
culturais, culminou com fenômenos de divergência entre gerações, tais como a revolução sexual, o surgimento de consumos culturais tipicamente juvenis e a participação política dos estudantes secundaristas e universitários. O tempo livre desfrutado pelos jovens passou a despertar a preocupação da sociedade adulta e levou à elaboração de uma série de programas esportivos, recreativos e campanhas preventivas de saúde para afastá-los das condutas socialmente reprovadas, como é o caso do consumo de drogas e do livre exercício da sexualidade (Abad, 2002).

Cabe relacionar à mudança de condição social do jovem, iniciada a partir da segunda década do século XX, as modalidades de políticas públicas destinadas aos adolescentes e aos jovens, elaboradas pelos governos nacionalistas e populistas da América Latina, incluindo o brasileiro, cujos interesses eram a modernização das sociedades latino-americanas, a substituição das importações e o desenvolvimento das indústrias nacionais, traços típicos de uma economia em expansão e com vasta disponibilidade de crédito externo. Em seguida, a partir da década de 1970, as políticas são centradas no controle social dos setores juvenis mobilizados, e isso por meio de doutrinação e de ações repressivas por parte do Estado às manifestações estudantis inconformistas. Esse caráter violento e opressor das intervenções do Estado chegou ao auge com a suspensão das garantias constitucionais e o incremento das restrições às liberdades civis (Abad, 2002).

Ao mesmo tempo em que o jovem é percebido como ator social, protagonista de movimentos em busca da restauração da democracia nos países que viviam sob os regimes ditatoriais, também era foco de intensas preocupações por parte do mundo adulto, o que despertou no Estado a atenção para a questão da juventude. Assim, ao se analisarem as políticas públicas destinadas à adolescência e juventude no Brasil, percebe-se que são estruturadas a partir dos conflitos entre os jovens e adolescentes, de um lado, e a sociedade adulta, de outro, configurando-se a questão como um problema de ordem política. De forma geral, a história das políticas voltadas para os jovens de toda a América Latina se reveste de um caráter setorial ou focalizado, cujas ações recaem sobre as camadas mais pauperizadas da sociedade, o que as distingue de outras iniciativas sociais do Estado, ditas universais, como as políticas de educação e saúde.

Do ponto de vista biológico, percebem-se, por um lado, a antecipação da puberdade, prolongando a adolescência em muitos anos ${ }^{2}$, e, por outro, a dissociação entre a maturidade sexual e a capacidade de emancipação, o que leva à emergência de um contingente de pessoas sexualmente maduras, embora ainda em condições físicas, emocionais, intelectuais e profissionais socialmente caracterizadas como incompletas ou insuficientes.

Vinculada ao prolongamento do período de adolescência, há uma mudança de expectativas da sociedade em relação à juventude, que passa a ser vista não como condição transitória, mas como valor em si. É o que se denomina processo de juvenilização da sociedade, gerando transformações no ciclo da vida (Castro, 10 jun. 2008). Tomem-se como exemplo o esporte, as vestimentas, os cuidados com o corpo, que ressaltam o despertar da juventude como um bem social. O processo de envelhecimento, tido até então como o período em que o passar dos anos trazia mais poder, influência e riqueza, causa, agora, insatisfação.

A nova cultura juvenil das sociedades urbanas, surgida na segunda metade do século $\mathrm{XX}$, deixa de ser a marca de um período de transição e passa a se configurar como a etapa 
final do pleno desenvolvimento humano; torna-se dominante nas economias de mercado desenvolvidas e se internacionaliza ou globaliza. Essa cultura e a categoria social a ela associada - os jovens - só poderiam ter surgido num contexto urbano de afluência industrial, no qual ocorreram ampliação da expectativa de vida, retardamento do ingresso no mercado de trabalho, maior especialização profissional, escolarização ampliada e crescente complexidade da divisão social do trabalho. Os filhos deixaram de ser mão de obra imprescindível no contexto familiar (nas camadas médias e altas), a cultura tornou-se mais secularizada, e as formas tradicionais de controle social foram contestadas, dissolvidas e/ou abrandadas.

\section{O nascimento da medicina de adolescentes}

A medicina de adolescentes se originou, primordialmente, do interesse de um grupo de médicos atuantes em escolas para rapazes na Inglaterra. Em 1884 se constitui a Medical Officers of Schools Association, primeira entidade organizada com o objetivo de reunir médicos em escolas, para debater assuntos e trocar experiência a respeito de regras e condutas ideais para um bom atendimento clínico aos jovens estudantes (Gallagher, 1982).

A partir de então, alguns temas - por exemplo, crescimento, desenvolvimento e questões relacionadas aos distúrbios de aprendizagem, como a dislexia - foram investigados, e os resultados, publicados em revistas médicas inglesas. Percebe-se nitidamente a emergência de questões vinculadas aos cuidados preventivos dirigidos aos jovens, de forma a minimizar os riscos de aquisição de doenças, prevenir acidentes e melhorar a qualidade do ambiente escolar, aspectos que constituíram a força motriz para a organização de uma área médica cujo objetivo é assistir e orientar, do ponto de vista clínico, o processo da adolescência (Gallagher, 1982).

Após a Primeira Guerra Mundial, surgiram os primeiros serviços médicos para estudantes nas grandes universidades e escolas militares dos EUA. Com isso, assuntos como os cuidados de saúde e bem-estar foram-se disseminando pelo país e se tornaram de interesse público. Ao longo da década de 1930, universidades norte-americanas como Yale e Harvard organizaram serviços de saúde para o atendimento de seus estudantes, o que levou a um melhor conhecimento a respeito dos adolescentes, de seus processos normais fisiológicos e psicológicos, e ao reconhecimento de suas diferenças em relação à criança e ao adulto, possibilitando, ademais, a melhoria dos cuidados e da prevenção de doenças (Gallagher, 1982).

As primeiras publicações de peso sobre a adolescência remontam ao início do século XX, a partir do clássico tratado de G. Stanley Hall, de 1904, Adolescência: sua psicologia e sua relação com a fisiologia, sociologia, sexo, crime, religião e educação, que influenciou uma geração de profissionais, marcou os estudos sobre a adolescência e, de certa forma, legitimou esse campo de estudo e atenção (Silber, 1997). Em 1918 foi publicado o primeiro artigo científico médico sobre o adolescente: "O trabalho da Clínica de Adolescentes da Faculdade de Medicina da Universidade de Stanford" (citado em Silber, 1997), no qual a autora (Amélia Gates) já apresenta, além dos problemas médicos atribuídos aos adolescentes, muitos dos princípios das atuais propostas de medicina de adolescentes.

As primeiras equipes científicas destinadas a estudar alguns problemas biológicos da adolescência (desenvolvimento, nutrição e endocrinologia da puberdade) foram formadas nas décadas de 1920 e 1930, sendo o grupo mais antigo o da Unidade de Estudos de Adolescentes 
da Universidade de Yale, integrado por anatomistas, fisiologistas, químicos, biólogos, pediatras e psicólogos (Silber, 1997). Cabe lembrar que muitas vezes tais investigações eram extensões de estudos iniciados com crianças. Em 1942, em Viena, Greulich et al. publicaram um trabalho fundamental, Estudos somáticos e endocrinológicos do rapaz púbere, no qual se estabeleceram os níveis de maturação sexual, cuja sistematização, elaborada por Tanner (citado em Silber, 1997), permitiu a existência de uma linguagem comum a pediatras, ginecologistas, endocrinologistas e clínicos gerais com relação ao desenvolvimento sexual.

Gallagher (1982), Alderman, Rieder e Cohen (2003) e outros autores norte-americanos, ao analisar o contexto histórico-cultural no qual se organizaram os primeiros serviços de medicina de adolescentes do início do século XX, referem-se à emergência de problemas sociais como a violência juvenil, o desemprego e as desigualdades econômicas, nos EUA e em outros países, como fatores associados à problemática de saúde do adolescente. Assim, questões como morbidade e mortalidade juvenis passaram a ser alvo de estudo e debate naquele momento: o impacto de novos comportamentos entre adolescentes, como o tabagismo; a discussão sobre sexualidade; violência; lesões causadas por esporte; avanços tecnológicos em relação à contracepção; doenças crônicas; saúde mental e psicofarmacologia na adolescência. Tudo isso contribuiu para a formação de uma nova especialidade clínica.

Em 1938 a Academia Americana de Pediatria incluiu, pela primeira vez, o termo adolescência em seus programas, e em 1951 houve a formação da primeira Unidade de Adolescentes nos Estados Unidos, no Boston Children's Hospital, sob o comando do médico J. Roswell Gallagher, que publicou, em 1960, o livro $A$ atenção médica ao adolescente, o primeiro compêndio sobre medicina de adolescentes.

Estabeleceu-se em 1968 a Society for Adolescent Medicine (SAM), cujo objetivo era incrementar a atenção médica para adolescentes e jovens. A atividade dos membros da SAM, como a de seus vários comitês, proporcionou a publicação regular de um boletim informativo, que deu origem, em 1980, ao Journal of Adolescent Health Care. A partir de então, outras revistas especializadas foram surgindo, como Adolescence, International Journal of Adolescent Medicine and Health, The Journal of Adolescent Research, The Journal of Pediatrics and Adolescent Gynecology (Saito, 2005).

Na América Latina, o serviço pioneiro de medicina de adolescentes foi fundado em 1958, em Buenos Aires, Argentina, sob a coordenação da médica Nydia Gomes Ferrarotti (Crespin, 2007). Em seguida, surgiram outros: no Chile, sob a coordenação de Paula Pelaz, e o de Enrique Dulanto, no México, ambos em 1964 (Crespin, 2007).

\section{A medicina de adolescentes no Brasil: o contexto inicial no estado de São Paulo}

As propostas pioneiras voltadas especificamente para a medicina de adolescentes no Brasil apareceram em São Paulo e no Rio de Janeiro, no início da década de 1970, vinculadas a faculdades de medicina e hospitais universitários. Em fevereiro de 1974, o primeiro serviço de medicina de adolescentes foi implantado sob a supervisão de Anita Colli, na disciplina de Pediatria Preventiva e Social do Departamento de Pediatria do Hospital das Clínicas da Faculdade de Medicina da Universidade de São Paulo (HC/FMUSP). Essa inserção valorizava aspectos preventivos que motivaram a atenção integral à saúde do adolescente. Em julho de 
1974, Evelyn Eisenstein e Maria Helena Ruzany foram as responsáveis pela implantação da Unidade Clínica para Adolescentes no Hospital Universitário Pedro Ernesto, da Universidade Estadual do Rio de Janeiro (Uerj). Em 1975 foi constituído o terceiro serviço brasileiro de medicina de adolescentes, a Clínica de Adolescência do Departamento de Pediatria da Faculdade de Ciências Médicas da Santa Casa de São Paulo, sob a coordenação de Verônica Coates.

Antes da década de 1970 não havia no Brasil médicos e serviços de saúde destinados especificamente para os adolescentes, embora, naturalmente, os médicos já atuassem, em clínicas particulares e nos serviços públicos, no atendimento de adolescentes. De acordo com a queixa ou problemática apresentada, o jovem podia dirigir-se a um clínico geral ou pediatra; adolescentes do sexo feminino consultavam-se com ginecologistas quando as questões referentes à saúde reprodutiva e sexual estavam em pauta, ficando, nesse âmbito, os do sexo masculino negligenciados pela assistência médica (Colli, Deluqui, 1989).

O atendimento dos adolescentes no Hospital das Clínicas da FMUSP contava com uma equipe constituída por médico, assistentes sociais, enfermeiros e nutricionistas, alicerçada nos princípios de promoção, proteção e recuperação da saúde, à luz de uma visão global do adolescente. A expressão atenção global à saúde era utilizada naquele momento para se referir às características de crescimento e desenvolvimento físico, problemas de saúde e relações do adolescente com o meio ambiente; buscava-se, portanto, integrar os propalados aspectos biopsicossociais (Colli et al., 1979). Em 1976 houve o ingresso de mais um médico na equipe, a doutora Maria Ignez Saito, que assumiu sua chefia em 1995.

Médicos de todo o Brasil foram recebidos na Unidade de Adolescentes do Instituto da Criança do HC/FMUSP para estágios de médico observador e de complementação especializada, muitos dos quais foram responsáveis pela implantação de serviços de atendimento aos adolescentes em todo o país, como os de Goiânia, Vitória, Botucatu, Florianópolis, Marília, Taubaté, São José do Rio Preto, entre outros, ligados às universidades locais (Saito, 2005).

A Faculdade de Ciências Médicas da Universidade Estadual de Campinas (Unicamp) contribuiu significativamente para a constituição do campo da saúde do adolescente no país, em especial por meio dos Departamentos de Tocoginecologia, Psiquiatria e Pediatria, que congregavam profissionais ativamente participantes da formação e divulgação do saber médico sobre o adolescente. Dentre eles, destaca-se Maurício Knobel, renomado psiquiatra e psicanalista formado em Buenos Aires, autor, em parceria com Arminda Aberastury, da obra Adolescência normal, lançada originalmente em 1973, marco da visão psicanalítica no entendimento da crise da adolescência.

Ao mesmo tempo que Knobel e seus alunos Everardo Buoncompagno e Fabio Adamo implantavam os serviços de psiquiatria da adolescência, o tocoginecologista João Luiz de Carvalho Pinto e Silva instituía o ambulatório de pré-natal de adolescentes. A iniciativa era já resultado de sua percepção quanto à necessidade de atendimento específico a essa faixa etária, diante da vivência assistencial a essa população, e da influência recebida dos profissionais do Instituto da Criança e da Santa Casa de São Paulo, por ocasião de congressos e capacitação sobre a adolescência.

Cabe ressaltar a relevância do serviço de atenção à saúde do adolescente vinculado à Uerj no cenário nacional da implantação de programas de saúde destinados a essa população e no 
tocante ao desenvolvimento de pesquisa e ensino no ambiente acadêmico (devido, aliás, às parcerias estabelecidas com as instituições universitárias paulistas), ainda que não se configure como objetivo deste artigo resgatar a atenção aos adolescentes no estado do Rio de Janeiro.

A partir da análise dos primeiros trabalhos brasileiros sobre a adolescência e a saúde dos adolescentes, percebe-se o desencadeamento de ações e propostas para a institucionalização tanto da medicina de adolescentes quanto de programas de saúde coletiva visando à saúde dos adolescentes nos moldes do que preconizavam as instituições pioneiras nesse tipo de atendimento. Cumpre assinalar que, na maioria dos serviços universitários, a atenção à saúde do adolescente já merecia abordagem integral, pautada pelo modelo das equipes multiprofissionais e interdisciplinares.

Outro aspecto relevante na implantação da medicina de adolescentes no país foi o diálogo entre instituições universitárias como USP, Uerj, Unicamp e, posteriormente, outras, quando barreiras foram rompidas, favorecendo a divulgação de diferentes experiências e saberes integrados a um objetivo único. Há, entretanto, particularidades nas perspectivas em relação à saúde do adolescente adotadas pela clínica e pela saúde coletiva, presentes no momento da institucionalização da medicina de adolescentes no estado de São Paulo, e pelas políticas públicas de saúde voltadas para essa faixa etária, o que será abordado adiante.

As organizações de saúde (Opas, OMS e Ministério da Saúde) também dialogaram com as instituições universitárias para estabelecer as diretrizes e normas dos programas de atendimento aos adolescentes no Brasil. Foi fruto dessa aproximação o Seminário Latinoamericano sobre a Saúde do Adolescente, organizado pela Opas e pelo Ministério da Saúde, no Rio de Janeiro em 1977. Essa interlocução torna-se ainda mais presente com o Curso de Adolescência promovido pelo Ministério da Saúde - Divisão Materno-infantil, realizado pelo Instituto da Criança, Departamento de Pediatria da FMUSP e Departamento de Psiquiatria da Unicamp, em 1981. Já com o objetivo de capacitação multiprofissional, foram realizados, em 1993, 1994 e 1995, os Cursos de Aprimoramento em Crescimento, Desenvolvimento e Nutrição na Adolescência, promovidos pela Unidade de Adolescentes do Instituto da Criança - já então Centro de Referência Opas/OMS - do Departamento de Pediatria da FMUSP. Outros serviços ligados a universidades, hospitais e centros de saúde da rede pública começaram a multiplicar-se pelo Brasil durante a década de 1980 (Saito, 2005).

Ao longo da trajetória da implantação dos serviços de atenção à saúde do adolescente, foram-se constituindo também os comitês científicos vinculados à Sociedade Brasileira de Pediatria, que mais tarde acabaram sendo substituídos por seus departamentos.

Em 1985, na cidade de São Paulo, ocorreu o Primeiro Congresso Brasileiro de Adolescência. Esse congresso, presidido por Anita Colli, vice-presidido por Maria Helena Ruzany e secretariado por Maria Ignez Saito, representou um marco na trajetória da Medicina de Adolescentes no país, pelo reconhecimento da importância do assunto, sobretudo entre os pediatras. Até então, os cursos sobre adolescência eram ministrados durante os congressos de Pediatria. A partir desse primeiro evento, o modelo de atenção integral à saúde do adolescente ganhou destaque tanto no âmbito da Sociedade Brasileira de Pediatria (que inclui os congressos de adolescência em sua agenda de realizações até os dias de hoje, nos quais a prova para o Título de Habilitação em Medicina de Adolescentes é aplicada), quanto no panorama internacional de atenção à saúde dessa faixa etária, visto que em 2001, durante o Oitavo Congresso 
Brasileiro de Adolescência realizado na Bahia, o Brasil sediou o Sétimo Congresso Internacional de Adolescência (Saito, 2005).

Em 1987 tiveram início as atividades do Programa de Saúde do Adolescente da Secretaria de Estado da Saúde, coordenado pela ginecologista Albertina Duarte Takiuti. O programa foi elaborado a partir de uma produção teórico-prática da comissão científica constituída por representantes de universidades paulistas: da Unicamp, o obstetra e ginecologista João Luiz Pinto e Silva, que a presidia, os psiquiatras Maurício Knobel e Everardo Buomcompagno, e a psicóloga Regina Célia Sarmento; da USP, as pediatras Anita Colli e Maria Ignez Saito, os obstetras e ginecologistas Albertina Duarte Takiuti e Álvaro de Cunha Bastos; da Universidade Federal Paulista, Sérgio Tufik, e da Santa Casa de São Paulo, Verônica Coates. Essa comissão científica apoiou o Grupo Executivo do Programa de Adolescentes (Gepro) na implantação de programas de saúde para adolescentes nas diversas regiões do estado de São Paulo, promovendo a capacitação e reciclagem de profissionais no âmbito da rede básica de saúde, enfatizando aspectos da atenção primária e da prevenção para essa faixa etária (Saito, 2005). Trata-se de exemplo claro do fortalecimento da relação docente-assistencial, por meio do conhecimento acadêmico levado à rede básica de saúde, que se concretizou pela produção de diversos materiais didáticos, além das importantes publicações Adolescência e saúde (São Paulo, 1988, 1994b, 1999b); Adolescência e Saúde 2 (1994a, 1999a).

Em 1989 por meio da Divisão Materno-infantil, o Ministério da Saúde incluiu a assistência primária à saúde dos adolescentes em suas atribuições. Com esse intuito, foram elaborados programas de atendimento ao adolescente, fundamentados na política de promoção de saúde, identificação de grupos de risco, detecção precoce dos agravos, tratamento adequado e reabilitação. Profissionais de saúde de vários estados e com experiência em adolescentes elaboraram normas técnicas para o atendimento de indivíduos dessa faixa etária, assim como realizaram treinamentos de sensibilização e de capacitação de equipes multiprofissionais, e formação de adolescentes como agentes multiplicadores de saúde e distribuição de material educativo (Coates, 1999).

Também em 1989, foi criado o Programa de Saúde do Adolescente (Prosad), vinculado à Divisão Materno-infantil do Ministério da Saúde, coordenado por Rachel Niskier Sanchez. O objetivo do Prosad era proporcionar acesso do adolescente ao sistema de saúde via atenção primária, com enfoque na promoção de saúde e prevenção de agravos, inserido na proposta hierarquizada do SUS e pautado na atenção integral por meio de abordagem multiprofissional. A partir de 1993, passou a ser denominado Serviço de Assistência à Saúde do Adolescente (Sasad). Nessa ocasião, editou-se a primeira publicação oficial do programa, Normas de atenção à saúde integral do adolescente (Brasil, 1993), que estipulou as diretrizes gerais para o atendimento de adolescentes e contemplou assuntos do crescimento e desenvolvimento na adolescência, os distúrbios da puberdade e o desenvolvimento psicológico do adolescente. O enfoque dessa primeira publicação do Prosad, elaborada por equipe constituída por membros do Ministério da Saúde e da Opas (coordenadores), profissionais vinculados às secretarias estaduais e docentes das universidades brasileiras, privilegia a concepção de adolescência como período de risco para a saúde e o processo de adoecimento relacionado às precariedades ambientais e ao contexto socioeconômico. Para o enfrentamento da problemática de saúde apresentada pelos adolescentes, são estabelecidas como prioridade a abordagem dos aspectos 
nutricionais e a monitorização do crescimento e desenvolvimento nas unidades de atenção primária (Brasil, 1993).

A Associação Brasileira de Adolescência (Asbra) foi fundada em 1989 por um grupo de profissionais interessados na saúde dos adolescentes, sendo Verônica Coates a primeira presidente eleita. A Asbra e suas regionais favoreceram a promoção, solidificação, normatização e regulação da prática profissional, o que, de certa forma, contribui para sua legitimação e seu reconhecimento, envolvendo também profissionais não médicos.

Cabe lembrar que a implantação da saúde do adolescente nas principais universidades do país ocorreu durante período político extremamente difícil e antidemocrático, o da ditadura militar, que durou de 1964 a 1985. Em 1974, ano da institucionalização da medicina de adolescentes em São Paulo e no Rio de Janeiro, o país vivia sob o AI-5, considerado o mais autoritário dos atos institucionais, que decretava a suspensão de muitos direitos políticos dos cidadãos.

Em meio a esse quadro ditatorial que impedia o cidadão de exercer seu papel pelas vias democráticas, por meio do voto e das manifestações populares, os jovens da sociedade brasileira (principalmente os estudantes do ensino médio e de nível superior) organizavam-se para reivindicar o fim da ditadura militar no país. Os movimentos estudantis ganharam força e incitaram numerosas manifestações de protesto contra o sistema vigente - encabeçadas por políticos de esquerda, intelectuais, professores e alunos universitários -, violentamente reprimidas pelo governo.

\section{A integralidade como princípio norteador da atenção à saúde dos adolescentes}

No estado de São Paulo, o conhecimento produzido por pesquisadores, professores e pela equipe multiprofissional das grandes universidades foi responsável pelo desenvolvimento de toda a rede de atenção à saúde pública para os adolescentes.

Percebe-se, ademais, a preocupação, ressaltada nos primeiros trabalhos sobre o tema, de delimitar uma área, um campo de atuação, e, para isso, circunscrever, por meio de sólida base teórica, as questões clínicas, o tipo de atendimento e, principalmente, a compreensão do adolescente à luz da saúde integral e da prevenção de agravos. Estabelecidos tais objetivos, tornou-se fundamental configurar a equipe multidisciplinar, único modelo capaz de atender às necessidades e demandas de saúde referentes a essa população.

Quanto ao atendimento médico, já enfatizavam Colli et al. em 1979 que, apesar de se conservarem os padrões tradicionais de elaboração da anamnese e da realização do exame físico, o adolescente e o responsável eram entrevistados separadamente pelo médico, e o conhecimento dos fatores ambientais que influenciavam a saúde do adolescente propiciavam a ampliação dos diagnósticos estabelecidos. Em publicações posteriores da equipe, conforme destaca Colli (1985), evidenciou-se ainda mais categoricamente a valorização da atenção global à saúde, nitidamente ameaçada pela cisão entre os aspectos físicos e psicossociais do adolescente, o que levaria a abordagens unilaterais e a resultados insatisfatórios.

Essa prerrogativa do anseio pela medicina integral se afirmava em época na qual a especialização médica se fortalecia e, com ela, a tendência progressiva ao reducionismo da 
patologia e ao olhar fragmentado do especialista, conhecedor em profundidade de um órgão ou sistema, mas, contudo, carente da compreensão do ser humano como um todo.

A consulta do adolescente configurou-se como uma das questões fundamentais para a obtenção de sucesso na abordagem médica e da equipe multiprofissional devotada a essa população, que apresentava formas peculiares tanto de procura dos serviços de saúde quanto de adoecimento. As estatísticas de serviços de atenção primária que atendiam adolescentes indicam que a morbidade por eles apresentada, com exceção do principal motivo de consulta, constituído por questões vinculadas à gravidez, relacionava-se principalmente ao grupo das chamadas queixas gerais e inespecíficas, traduzidas em geral no diagnóstico de problemas de caráter psicoemocional (Ayres, 1990).

Estabelecer os aspectos éticos implicados na especificidade da consulta dessa faixa etária foi outro aspecto fundamental para consolidar a assistência à saúde como dispositivo para detectar riscos e realizar prevenção na adolescência.

Passou-se a preconizar que preceitos éticos de atendimento de saúde ao adolescente se deviam aplicar a todos os profissionais da equipe, sendo explicitados logo na primeira entrevista com o paciente e sua família, sobretudo a garantia do sigilo de suas informações, assim como seus limites, isto é, a quebra de sigilo, que ocorre com o conhecimento prévio do adolescente, em função de situações ou diagnósticos por ele apresentados.

As primeiras publicações brasileiras a respeito do atendimento do adolescente e seus aspectos éticos, que compreendem o sigilo, a privacidade e a confidencialidade, remontam ao final da década de 1970 e ao início da seguinte. Por sigilo compreende-se o segredo médico assegurado ao adolescente pelo Código de Ética Médica. ${ }^{3}$

Segundo Saito (2005), o respeito à privacidade e à confidencialidade é pressuposto para a assistência de qualidade no atendimento ao adolescente, que deve garantir além disso a abordagem da saúde sexual e reprodutiva, do uso abusivo de drogas e da violência em suas diversas formas, como abuso sexual, maus-tratos, agressão, negligência etc.

A evolução dos aspectos éticos referentes à consulta do adolescente teve início com o Código de Ética Médica, que durante quase duas décadas foi a única proteção legal ao atendimento médico do adolescente segundo os princípios da privacidade e confidencialidade. Esse código foi seguido, posteriormente, pelo ECA, ao garantir o direito dos adolescentes à saúde, à liberdade, ao respeito e à dignidade. ${ }^{4}$

Os médicos pioneiros no atendimento de adolescentes, sobretudo os pediatras, já apontavam a existência de lacuna na atenção à saúde aos indivíduos dessa faixa etária, e a atribuíam em parte à falta de motivação e de preparo dos profissionais para a consulta com o adolescente, assim como seu temor pelos aspectos legais e éticos (Rossi et al., 1983).

\section{Atenção integral à saúde dos adolescentes: a perspectiva da clínica e da saúde coletiva}

Ao se analisar a institucionalização da medicina de adolescentes no Brasil, sobretudo no estado de São Paulo, percebe-se que, enquanto a abordagem clínica se defronta com os desafios de delimitar um campo de trabalho - estabelecer metodologia de atendimento, modelos de consulta pautados no indivíduo como paciente, e, portanto, garantir-lhe momentos de 
privacidade e confidencialidade, além de definir os diversos aspectos de normalidade física, o que permite identificar o que seria normal e patológico, integrando prevenção e tratamento -, a saúde coletiva, por sua vez, lança olhar mais abrangente sobre esses aspectos, buscando situá-los em uma perspectiva socioepidemiológica.

A fim de ilustrar o enfoque da saúde coletiva, organizada em torno da noção de programação em saúde, sobretudo com o objetivo de projetar modelos assistenciais alternativos e práticas de intervenção nos doentes e nas condições relativas à promoção da saúde da população, resgata-se o Programa de Atenção à Saúde do Adolescente (Pasa), instituído no Centro de Saúde Escola do Butantã (CSEB), no final da década de 1980, sob coordenação do médico sanitarista José Ricardo de Carvalho Mesquita Ayres, desenvolvido junto à Clínica Médica de Adultos e vinculado ao Departamento de Medicina Preventiva da USP. Instituído em 1989, o Pasa voltou-se para o atendimento de adolescentes de 12 a 18 anos da região do Butantã, mais precisamente, das favelas de São Remo e São Domingos. Partindo da crítica à leitura individualizada e universalista, que era a predominante nas abordagens clínicas da medicina em geral, apontava a necessidade de uma organização do trabalho de atenção à saúde que incorporasse as dimensões sociais e coletivas da adolescência concretamente experimentadas em cada contexto de atenção à saúde (Ayres, 1990).

A fim de se compreender esse movimento no âmbito da saúde coletiva, faz-se necessário retomar, mesmo que sucintamente, o movimento da reforma sanitária iniciado no final da década de 1960 e que deu origem a esse campo.

O conceito de saúde coletiva que emerge a partir do final da década de 1970 recebe influências dos movimentos anteriores a esse período, entre eles o projeto preventivista, que se inicia na segunda metade da década de 1950, especialmente nos países latino-americanos. Esses países identificavam um momento de crise do modelo médico tradicional, que suscitou respostas na formação médica na década de 1960. As críticas que se apontavam à maneira organicista e 'biologizante' de atenção médica, centrada em práticas individuais e com base no hospital, geraram reformas que se concretizaram, em um primeiro momento, no plano pedagógico, sem, no entanto, incitar mudanças nas práticas médicas.

A integração tão citada do biopsicossocial teve aqui suas origens, e o modelo de medicina integral, apropriado da expressão norte-americana comprehensive medicine, aparece na versão latino-americana, especialmente na esfera da medicina de família integrada à comunidade. Se no aspecto pedagógico o movimento preventivista acrescenta ao campo da saúde conceitos das áreas das ciências humanas, no plano político-ideológico tais concepções são manifestadas junto à prática da chamada medicina comunitária e seus desdobramentos nos programas extramuros (Nunes, 1994).

A trajetória histórica e conceitual da saúde coletiva recebeu influências de ordem socioeconômica, medidas que se vinham desenrolando desde a segunda metade do século XX, culminando, na década de 1980, na chamada nova ordem mundial, inspirada no neoliberalismo, preconizador da lei do estado mínimo e do corte nos gastos públicos como resposta à crise fiscal do Estado. Isso tudo prejudicou o desenvolvimento e a implantação das propostas de saúde pública que vinham sendo discutidas desde as décadas de 1960 e 1970, como o direito à saúde, a extensão de cobertura dos serviços de saúde e o reconhecimento da importância da sociedade em garantir os cuidados básicos de saúde. Nesse sentido, a saúde 
pública dos países capitalistas dependentes passou a enfrentar um período de crise, uma vez que todos os esforços visando à universalização da saúde, sintetizados no lema da OMS "Saúde para todos no ano 2000", e à valorização da atenção primária à saúde como estratégia para obtenção desses objetivos, difundidos a partir da Conferência de Alma-Ata, em 1978 (Declaração..., 1978), ficaram dificultados pelo modelo econômico que passou a privilegiar o mercado como mecanismo para a alocação de recursos e questionar a responsabilidade estatal na provisão de bens e serviços para o atendimento de necessidades sociais, entre elas, a saúde.

Diante desse cenário, emerge a necessidade de novas discussões sobre a questão da saúde na perspectiva público-coletivo-social e as subsequentes propostas de ação, o que contribuiu, sobretudo na América Latina, para a instituição do campo da saúde coletiva, inserido no movimento de renovação tanto da teoria quanto da prática da saúde pública. Por meio da constituição desse novo campo, visualiza-se a oportunidade para a efetiva incorporação de propostas centrais da renovação da saúde pública, como o complexo "promoção-saúdedoença-cuidado". Viabilizam-se assim as metas do 'Saúde para todos' mediante a adoção de políticas públicas com a participação da sociedade nas questões de vida, saúde, sofrimento e morte (Paim, Almeida Filho, 1998).

A grande questão ou foco de tensão dessa nova concepção de saúde era a maneira como esses saberes se aplicavam à prática médica, à assistência à saúde, à elaboração de políticas públicas e à gestão dos serviços e programas para os grupos populacionais. A área clínica, ainda que considerasse as influências externas ao processo de saúde e doença, mantinha seu olhar centrado na singularidade do indivíduo, aspecto inerente a seu campo de atuação. Daí as dificuldades de interlocução entre essas duas disciplinas, e o embate acadêmico em torno da institucionalização de certas áreas, implantação de núcleos de estudo e pesquisa e de programas de saúde, sobretudo os de âmbito público.

\section{Considerações finais}

Essas breves incursões à história mostram-nos aspectos bastante relevantes para compreender algumas das configurações atuais e os desafios da saúde do adolescente como, simultaneamente, núcleo de expertise e campo de interesse na atenção à saúde.

Destaque-se, em primeiro lugar, o contexto mundial e brasileiro, pano de fundo do desenvolvimento da atenção à saúde do adolescente nos anos 1970. De fato, se a adolescência e a juventude são criação das sociedades ocidentais oitocentistas, foi apenas em meados do século XX que seu papel social atingiu talvez o momento de maior transcendência, interpelando fortemente áreas que, como a medicina, são extremamente sensíveis às exigências de respostas normativas às necessidades sociais. Assim, o saber da medicina moderna, representado hegemonicamente pela clínica, foi chamado a organizar nesse período mais uma área de saberes e práticas definidoras e construtoras de padrões de aceitação social (normalidade) cientificamente embasados.

Contraditoriamente, porém, essa nova área de atuação médica, plasmada sobretudo na pediatria, construiu-se em larga medida sobre a negação do caráter fragmentário predominante nas especialidades clínicas. Compromissada politicamente com a inclusão social dos adolescentes, em especial os mais pobres, a medicina de adolescentes brasileira buscou, com 
frequência, uma visão integradora, que articulasse os fenômenos biológicos da puberdade a uma série de outras leituras sobre as diversas dimensões (cognitivas, emocionais, econômicas, culturais, éticas) que caracterizam as necessidades concretas de saúde do indivíduo nessa faixa etária.

De outro lado, no contexto da América Latina, e do Brasil em particular, a perspectiva sanitária da organização dos modelos de atenção à saúde também encontrou no segmento dos adolescentes e jovens incontornável desafio, dada a relevância e particularidade epidemiológica, social e política da saúde dos adolescentes nesses países. Nesse sentido, a saúde coletiva aproximou-se do movimento da medicina de adolescente ao colaborar, por outras vias e a partir de outros campos de expertise (epidemiologia, gestão de serviços de saúde, ciências sociais e humanas em saúde), com a construção da saúde do adolescente como campo de práticas.

É certo que, em um primeiro momento, esses diferentes núcleos de expertise experimentaram alguma reserva crítica e dificuldade de diálogo entre si. É fato, porém, que, tanto no plano federal como no do estado de São Paulo, as propostas programáticas que surgem no final dos anos 1980 parecem ir logrando estabelecer crescente e produtiva interação entre esses dois núcleos, em favor da fecundidade do campo da saúde do adolescente.

Progressos maiores nesse sentido parecem, não obstante, depender de que arranjos corporativos, dogmatismos disciplinares e pressões institucionais conservadoras não obstaculizem os diálogos interdisciplinares e intersetoriais aí iniciados, os quais têm possibilitado à atenção à saúde do adolescente e do jovem constituir-se em legítima e produtiva experiência de atenção integral à saúde de indivíduos e grupos populacionais no Sistema Único de Saúde brasileiro.

\section{NOTAS}

${ }^{1} \mathrm{O}$ antropólogo Van Gennep (1978), em seu livro sobre ritos de passagem, elabora o conceito de marginalidade, pois o neófito é posto à margem de sua sociedade, devendo nesse momento submeter-se às tarefas exigidas pelos mais velhos para que, ao final desse período de transição, possa ser incorporado novamente ao grupo, ocupando outro status social, o que garante sua permanência na vida social regular. Passar, portanto, é permanecer.

${ }^{2}$ Segundo Marcondes (1979, p.1), se for aceito o argumento de que as primeiras modificações já caracterizam a adolescência, quer sejam elas de natureza "bio" (puberdade), "psico" ou social, e que a adultícia estará instalada de modo pleno com o desaparecimento das últimas características da adolescência, poder-se-ia estender essa fase da vida dos 10 aos 25 anos.

${ }^{3}$ Conforme o capítulo IX, artigo 74 do Código de Ética Médica (Conselho Federal de Medicina, 17 set. 2009): "É vedado ao médico revelar sigilo profissional relacionado a paciente menor de idade, inclusive a seus pais ou representantes legais, desde que o menor tenha capacidade de discernimento, salvo quando a não revelação possa acarretar dano ao paciente."

${ }^{4}$ Segundo o Estatuto da Criança e do Adolescente, capítulo I, artigo 7o: "a criança e o adolescente têm direito à proteção à vida e à saúde, mediante a efetivação de políticas sociais públicas que permitam o nascimento e o desenvolvimento sadio e harmonioso, em condições dignas de existência" (Brasil, 13 jul. 1990). 


\section{REFERÊNCIAS}

ABAD, Miguel.

Las políticas de juventud desde la perspectiva de la relacion entre convivencia, ciudadania y nueva condicion juvenil. Última Década, Santiago. v.10, n.16, p.117-152. 2002.

ABERASTURY, Arminda; KNOBEL, Maurício. Adolescência normal: um enfoque psicanalítico. Porto Alegre: Artes Médicas. 1986.

ALDERMAN, Elizabeth Meller; RIEDER, Jessica; COHEN, Michael I.

The history of adolescent medicine. Pediatric Research, Philadelphia, v.54, n.1, p.137-147. 2003.

ARIÈS, Philippe.

História social da criança e da família. Rio de Janeiro: Guanabara Koogan. 1981.

AYRES, José Ricardo de Carvalho Mesquita. Adolescência e saúde coletiva: aspectos epistemológicos da abordagem programática. In: Schraiber, Lilia Blima (Org.). Programação em saúde hoje. São Paulo: Hucitec. p.139-182. 1990.

\section{BRASIL.}

Ministério da Saúde. Secretaria de Assistência à Saúde. Departamento de Assistência e Promoção à Saúde. Coordenação Materno-infantil. Serviço de Assistência à Saúde do Adolescente. Normas de atenção à saúde integral do adolescente. v.1. Brasília: Ministério da Saúde. 1993.

BRASIL.

Presidência da República. Lei n.8069 de 13 de julho de 1990. Dispõe sobre o Estatuto da Criança e do Adolescente e dá outras providências. Disponível em: http://www. planalto.gov.br/ccivil_03/leis/L8069.htm. Acesso em: 12 jan. 2011. 13 jul. 1990.

BRASIL.

Presidência da República. Ato Institucional n.5. Diário Oficial da União, Brasília (DF). Seção 1:1. 13 dez. 1968.

CAMPOS, Gastão Wagner de Souza.

Saúde pública e saúde coletiva: campo e núcleo de saberes e práticas. Ciência e saúde coletiva, Rio de Janeiro, v.5, n.2, p.219-230. 2000.

CASTRO, Ana Lúcia de.

Imagens do corpo e cultura de consumo. ComCiência. Campinas, n.99. Disponível em: http://www.comciencia.br/comciencia/?section= $8 \&$ \&edicao=36\&id=426. Acesso em 1 jul. 2008. 10 jun. 2008.

COATES, Verônica.

História brasileira da medicina do adolescente: comemorando 10 anos de Asbra. Adolescencia
Latinoamericana, Porto Alegre, v.1, n.4, p.260-265. 1999.

COLLI, Anita S.

$\mathrm{O}$ adolescente e a equipe de saúde. Comunicação, São Paulo, v.6, n.1, p.4-5. 1985.

COLLI, Anita S.; DELUQUI, Celina Guerra. In: Alcântara, Pedro de; Marcondes, Eduardo (Org.). Pediatria Básica. São Paulo: Sarvier. p.175-189. 1989.

COLLI, Anita S. et al.

Atendimento de adolescentes. In: Setian, Nuvarte; Colli Anita S.; Marcondes, Eduardo (Org.). Adolescência. São Paulo: Sarvier. p.99-106. 1979.

CONSELHO FEDERAL DE MEDICINA. Resolução CFM n.1.931, de 17 de setembro de 2009. Aprova o Código de Ética Médica. Diário Oficial da União, Brasília (DF), p.90-92. 24 set. 2009. 17 set. 2009.

CRESPIN, Jacques.

Breve história da medicina da adolescência. In: Crespin, Jacques; Reato, Lígia de Fátima Nóbrega. Hebiatria: medicina da adolescência. São Paulo: Roca. p.4-9. 2007.

DECLARAÇÃO...

Declaração de Alma-Ata. Conferência

Internacional sobre Cuidados Primários de Saúde, Alma-Ata, URSS, 6-12 Set. 1978. Disponível em: http://www.dhnet.org.br/direitos/sip/onu/saude/ almaata.htm. Acesso em: 15 fev. 2013. 1978.

FORACCHI, Marialice Mencarini.

A juventude na sociedade moderna. São Paulo: Livraria Pioneira. 1972.

GALLAGHER, James Roswell.

The origens, development, and goals of adolescent medicine. Journal of Adolescent Health Care, New York, v.3, n.1, p.57-63. 1982.

HOBSBAWM, Eric J.

Era dos extremos: o breve século XX - 1914-1991. São Paulo: Companhia das Letras. 1997.

MARCONDES, Eduardo.

Introdução ao estudo da adolescência In: Setian, Nuvarte; Colli, Anita S.; Marcondes, Eduardo. Adolescência. São Paulo: Sarvier. p.1-12. 1979.

MATTOS, Marco Aurélio Vannucchi L.; SWENSSON JUNIOR, Wagner Cruz.

Contra os inimigos da ordem: a repressão política do regime militar brasileiro (1964-1985). Rio de Janeiro: DP\&A. 2003.

MUUSS, Rolf.

Teorias da adolescência. Belo Horizonte: Interlivros. 1976. 
NUNES, Everardo Duarte.

Saúde coletiva: história de uma ideia e de um conceito. Saúde e Sociedade, São Paulo, v.3, n.2, p.5-21. 1994.

\section{OMS.}

Organización Mundial de la Salud. Necesidades la salud de los adolescentes. Informe técnico, n.609. Ginebra: OMS. 1977.

PAIM, Jairnilson S.; ALMEIDA FILHO, Naomar. Saúde coletiva: uma "nova saúde pública" ou campo aberto a novos paradigmas? Revista de Saúde Pública, São Paulo, v.32, n.4, p.299-316. 1998.

ROJAS, Diego Salazar.

Adolescência, cultura y salud. In: Maddaleno, Matilde et al. La salud del adolescente y del joven. Washington, DC: Organización Panamericana de la Salud, p.15-26. 1995.

ROSSI, Vicente de Paula et al.

Atendimento de adolescentes: informações dos comitês - Comitê de Adolescência da Sociedade de Pediatria de São Paulo. Revista Paulista de Pediatria, São Paulo, v.1, n.3. 1983.

RUZANY, Maria Helena; SZWARCWALD, Célia Landmann.

Oportunidades perdidas de atenção integral do adolescente: resultados de estudo-piloto. Adolescência Latinoamericana, Buenos Aires, v.2, n.1, p.26-35. 2000.

SAITO, Maria Ignez.

Memorial [Introdução - concurso para professor livre-docente do Departamento de Pediatria]. São
Paulo: Faculdade de Medicina, Universidade de São Paulo. 2005.

SÃO PAULO.

Secretaria de Estado da Saúde de São Paulo. Comissão de Saúde do Adolescente. Adolescência e Saúde 2. São Paulo. 1999a.

SÃO PAULO.

Secretaria de Estado da Saúde de São Paulo.

Comissão de Saúde do Adolescente. Adolescência e Saúde. São Paulo: Paris Editorial. 1999b.

SÃO PAULO.

Secretaria de Estado da Saúde de São Paulo.

Comissão de Saúde do Adolescente. Adolescência e Saúde 2. São Paulo: Paris Editorial. 1994a.

SÃO PAULO.

Secretaria de Estado da Saúde de São Paulo.

Comissão de Saúde do Adolescente. Adolescência e Saúde. São Paulo: Paris Editorial. 1994b.

SÃO PAULO.

Secretaria de Estado da Saúde de São Paulo. Comissão de Saúde do Adolescente. Adolescência e Saúde. São Paulo: Paris Editorial. 1988.

SILBER, Tomás José.

Medicina de la adolescência: una nueva subespecialidad de la pediatría y la medicina interna en la América del Norte. Adolescência Latinoamericana, Buenos Aires, v.1, n.1, p.11-15. 1997.

VAN GENNEP, Arnold.

Os ritos de passagem. Petrópolis: Vozes. 1978. 